\title{
Schlegelella aquatica sp. nov., a novel thermophilic bacterium isolated from a hot spring
}

\author{
Yi-Ju Chou, ${ }^{1}$ Shih-Yi Sheu, ${ }^{2}$ Der-Shyan Sheu, ${ }^{2}$ Jih-Terng Wang ${ }^{3}$ \\ and Wen-Ming Chen ${ }^{1}$ \\ 1,2Departments of Seafood Science ${ }^{1}$ and Marine Biotechnology ${ }^{2}$, National Kaohsiung Marine \\ University, No. 142, Hai-Chuan Road, Nan-Tzu, Kaohsiung City 811, Taiwan \\ ${ }^{3}$ Department of Biotechnology, Tajen University, Pingtung, Taiwan
}

Wen-Ming Chen

p62365@ms28.hinet.net
The genus Schlegelella was proposed by Elbanna et al. (2003) and belongs to the subgroup Rubrivivax of the Betaproteobacteria. The genus Schlegelella currently comprises a single species with a validly published name, Schlegelella thermodepolymerans. The two strains of $S$. thermodepolymerans (K14 ${ }^{\mathrm{T}}$ and DhA-71) (Elbanna et al., 2003) and an independently isolated bacterium, Caenibacterium thermophilum (N2-680 ${ }^{\mathrm{T}}$ ) (Manaia et al., 2003), were described simultaneously as gen. nov., sp. nov. However, after confirming their closer relationship, Lütke-Eversloh et al. (2004) reclassified C. thermophilum $\mathrm{N} 2-680^{\mathrm{T}}$ as a later synonym of $S$. thermodepolymerans. All of the strains of $S$. thermodepolymerans are moderately thermophilic (optimum growth at $45-50{ }^{\circ} \mathrm{C}$ ) and have been isolated from man-made habitats. The type strain of $S$. thermodepolymerans was isolated from activated sludge, whilst $C$. thermophilum N2$680^{\mathrm{T}}$ was isolated from an aerobic digester of municipal sludge.

The aim of the present study was to determine the taxonomic position of the isolate $\mathrm{wcfl}^{\mathrm{T}}$, which was isolated from a water sample (natural habitat) collected from a hot spring located in the Tainan area, southern Taiwan.

The GenBank/EMBL/DDBJ accession number for the 16S rRNA gene sequence of strain $\mathrm{wcfl}^{\top}$ is DQ417336.

A table detailing the chemical composition of the Guanzingling hot spring water sample is available as supplementary material in IJSEM Online.
The water sample was collected in July 2005 from a hot spring in Guanzingling located in southern Taiwan. The temperature of the spring water ranged from 60 to $65^{\circ} \mathrm{C}$ and the $\mathrm{pH}$ value was approximately 8 . The chemical composition of the Guanzingling hot spring water sample is listed in Supplementary Table S1 in IJSEM Online. After diluting with sterile distilled water, the water sample was plated on $10 \%$ Luria-Bertani (LB) agar plates $(1.0 \mathrm{~g}$ tryptone, $0.5 \mathrm{~g}$ yeast extract, $1.0 \mathrm{~g} \mathrm{NaCl}$ and $15 \mathrm{~g}$ agar in 11 distilled water) and incubated at $55{ }^{\circ} \mathrm{C}$. A bacterial strain, designated $\mathrm{wcfl}^{\mathrm{T}}$, was isolated, maintained and subcultivated further on $10 \%$ LB medium. The organism was the subject of a polyphasic taxonomic study, which showed that it represented a novel species of Schlegelella.

Cell morphology was observed under a light microscope. The motility of cells was tested by the hanging-drop method. A Gram stain Set S (Difco) kit was used for testing the Gram reaction, and poly- $\beta$-hydroxybutyrate granule accumulation was observed by light microscopy after staining the cells with Sudan black. Flagellar staining was performed using a spot test flagella stain (Difco). The $\mathrm{pH}$ range for growth was determined by measuring $\mathrm{OD}_{595}$ of the culture grown in $10 \%$ LB broth that had been adjusted prior to sterilization to various $\mathrm{pH}$ values ( $\mathrm{pH} 3-11$ at intervals of $0.5 \mathrm{pH}$ units) using appropriate biological buffers (Chung et al., 1995). Growth at various temperatures $\left(15-70{ }^{\circ} \mathrm{C}\right)$ was measured on $10 \% \mathrm{LB}$ medium. Growth under anaerobic conditions was determined after incubation in an Oxoid AnaeroGen system. The performance of growth was recorded by measuring the $\mathrm{OD}$ of the culture with respect to time. 
Extraction of genomic DNA, PCR amplification and sequencing of the 16S rRNA gene were carried out as described previously (Chen et al., 2001). Sequence analysis was achieved using a DNA sequencer (ABI PRISM 310; Applied Biosystems) and sequence assembly by using the Fragment Assembly System program from the Wisconsin Package 9.1 supplied by the National Health Research Institute of Taiwan. The resultant sequence was compared with available 16S rRNA gene sequences in GenBank. Multiple-sequence alignment including strain $\mathrm{wcfl}^{\mathrm{T}}$ and its closest relatives was performed using BIOEDIT software (Hall, 1999). Phylogenetic trees were inferred using the maximumparsimony and neighbour-joining (Saitou \& Nei, 1987) tree-making algorithms. An evolutionary distance matrix was generated for the neighbour-joining algorithm using the Jukes \& Cantor (1969) distance model and bootstrap analysis (1000 resamplings).

For determination of $\mathrm{G}+\mathrm{C}$ content, a DNA sample was prepared and degraded enzymically into nucleosides as described by Mesbah et al. (1989). The obtained nucleoside mixture was then separated by HPLC. DNA-DNA hybridization experiments were performed with photobiotinlabelled probes as described by Ezaki et al. (1989). The degree of hybridization was determined from two replicate experiments. Further differentiation of strain $\mathrm{wcfl}^{\mathrm{T}}$ from its closest phylogenetic neighbour was determined by cellular fatty acid profiling using gas chromatography according to the instructions of the Microbial Identification System (MIDI; Microbial ID).

Strain $\mathrm{wcfl}^{\mathrm{T}}$ was examined for a broad range of phenotypic properties. Additional biochemical tests were performed at $50{ }^{\circ} \mathrm{C}$ using the Biolog GN2 (Biolog), API ZYM (bioMérieux) and API 20NE (bioMérieux) microtest systems according to the methods outlined by the manufacturers. Sensitivity to antibiotics was examined after spreading cells $(0.5 \mathrm{McF}$ arland) on $10 \% \mathrm{LB}$ medium and incubating at $50{ }^{\circ} \mathrm{C}$. Antibiotic discs (Difco) contained the following antibiotics: ampicillin $(10 \mu \mathrm{g})$, cefotaxime $(30 \mu \mathrm{g})$, chloramphenicol $(30 \mu \mathrm{g})$, gentamicin $(10 \mu \mathrm{g})$, kanamycin $(30 \mu \mathrm{g})$, nalidixic acid $(30 \mu \mathrm{g})$, novobiocin $(30 \mu \mathrm{g})$, rifampicin $(5 \mu \mathrm{g})$, penicillin $\mathrm{G}(10 \mu \mathrm{g})$, streptomycin $(10 \mu \mathrm{g})$ or tetracycline $(30 \mu \mathrm{g})$. The effect of antibiotics on cell growth was assessed after 3 days and susceptibility was scored based on the distance from the edge of clear zone to that of the disc. If the distance was larger than $3 \mathrm{~mm}$ the strain was classified as susceptible, between 1 and $3 \mathrm{~mm}$ it was classified as moderately susceptible and if the clear zone was less than $1 \mathrm{~mm}$ it was considered resistant.

Strain wcfl $^{\mathrm{T}}$ grew well aerobically in complex media with a reduced concentration of nutrients, such as $10 \% \mathrm{LB}$ medium. When higher concentrations of nutrients (such as trypticase soy, nutrient and LB medium) were provided, growth was comparatively poor. Strain $\mathrm{wcfl}^{\mathrm{T}}$ formed visible, cream or white colonies of circular and convex shape with entire edges. The colony size was approximately
$1.0-1.5 \mathrm{~mm}$ in diameter on $10 \% \mathrm{LB}$ agar plates after incubation for $48 \mathrm{~h}$ at $55^{\circ} \mathrm{C}$. Strain wcf1 ${ }^{\mathrm{T}}$ grew well at temperatures ranging from 30 to $60^{\circ} \mathrm{C}$ and from $\mathrm{pH} 6$ to 8 . Optimal growth occurred at $50^{\circ} \mathrm{C}$ and $\mathrm{pH} 7 \cdot 0$. Strain $\mathrm{wcfl}^{\mathrm{T}}$ did not grow after $120 \mathrm{~h}$ of incubation at $50{ }^{\circ} \mathrm{C}$ under anaerobic conditions.

Cells of strain $\operatorname{wcfl}^{\mathrm{T}}$ were Gram-negative rods, motile by means of polar flagellum, non-spore-forming, $0 \cdot 4-0 \cdot 5 \mu \mathrm{m}$ in diameter and $0 \cdot 8-2 \cdot 0 \mu \mathrm{m}$ in length. Poly- $\beta$-hydroxybutyrate granules were observed after staining with Sudan black.

A nearly complete $16 \mathrm{~S}$ rRNA gene sequence (1419 nt) was obtained for strain wcfl ${ }^{\mathrm{T}}$. A comparison of the sequence with those of representatives of genera classified in the Betaproteobacteria showed that the organism fell within the evolutionary radiation occupied by the genus Schlegelella (Fig. 1). According to the sequence similarity calculations, the organism was most closely related to $S$. thermodepolymerans strain $\mathrm{K}_{1}{ }^{\mathrm{T}}(97 \cdot 8 \%$ similarity) (Elbanna et al., 2003), S. thermodepolymerans strain N2-680 (97.9\% similarity) (Manaia et al., 2003), S. thermodepolymerans strain SA8 (97.9\% similarity), S. thermodepolymerans strain SA1 (97.9\% similarity) (Romen et al., 2004) and S. thermodepolymerans strain DhA-71 (97.8\% similarity) (Yu \& Mohn, 1999) (Fig. 1). The similarity levels of strain wcfl $^{\mathrm{T}}$ with other bacterial species with validly published names within the Betaproteobacteria were less than $95 \%$.

In the whole-genome DNA-DNA hybridization experiments, strain $\mathrm{wcfl}^{\mathrm{T}}$ showed a relatively low DNA-DNA relatedness value $(45 \cdot 0 \%)$ with $S$. thermodepolymerans $\mathrm{K} 14^{\mathrm{T}}$, clearly indicating that strain $\mathrm{wcfl}^{\mathrm{T}}$ represents a novel species.

The $\mathrm{G}+\mathrm{C}$ content of strain $\mathrm{wcfl}^{\mathrm{T}}$ was $69 \cdot 2 \mathrm{~mol} \%$, which is lower than that of $S$. thermodepolymerans $(70 \cdot 0 \mathrm{~mol} \%)$.

The cellular fatty acid composition of strain wcf1 ${ }^{\mathrm{T}}$ is shown in Table 1 in comparison with S. thermodepolymerans $\mathrm{K} 14^{\mathrm{T}}$. The fatty acid profile of strain $\mathrm{wcfl}^{\mathrm{T}}$ was similar to that of $S$. thermodepolymerans $\mathrm{K}_{1} 4^{\mathrm{T}}$, although the amounts of $16: 0$ and $17: 0$ cyclo differed. However, strain wcf1 ${ }^{\mathrm{T}}$ could clearly be distinguished from $S$. thermodepolymerans $\mathrm{K} 14^{\mathrm{T}}$ by the presence of a significant amount of summed feature 3 $(19 \cdot 5 \%)$.

In API 20NE tests, strain $\mathrm{wcfl}^{\mathrm{T}}$ was positive for oxidase (weak), nitrate reduction, aesculin hydrolysis, gelatin hydrolysis and assimilation of glucose, maltose, gluconate, adipate and phenyl acetate and negative for catalase, indole production, glucose fermentation, arginine dihydrolase, urease, $\beta$-galactosidase and assimilation of arabinose, mannose, mannitol, $\mathrm{N}$-acetylglucosamine, caprate, malate and citrate. In API ZYM tests, $\mathrm{wcfl}^{\mathrm{T}}$ was positive for alkaline phosphatase, C4 esterase, C8 lipase, C14 lipase, leucine arylamidase, naphthol-AS-BI-phosphohydrolase and $\alpha$ glucosidase and negative for valine arylamidase, cystine 


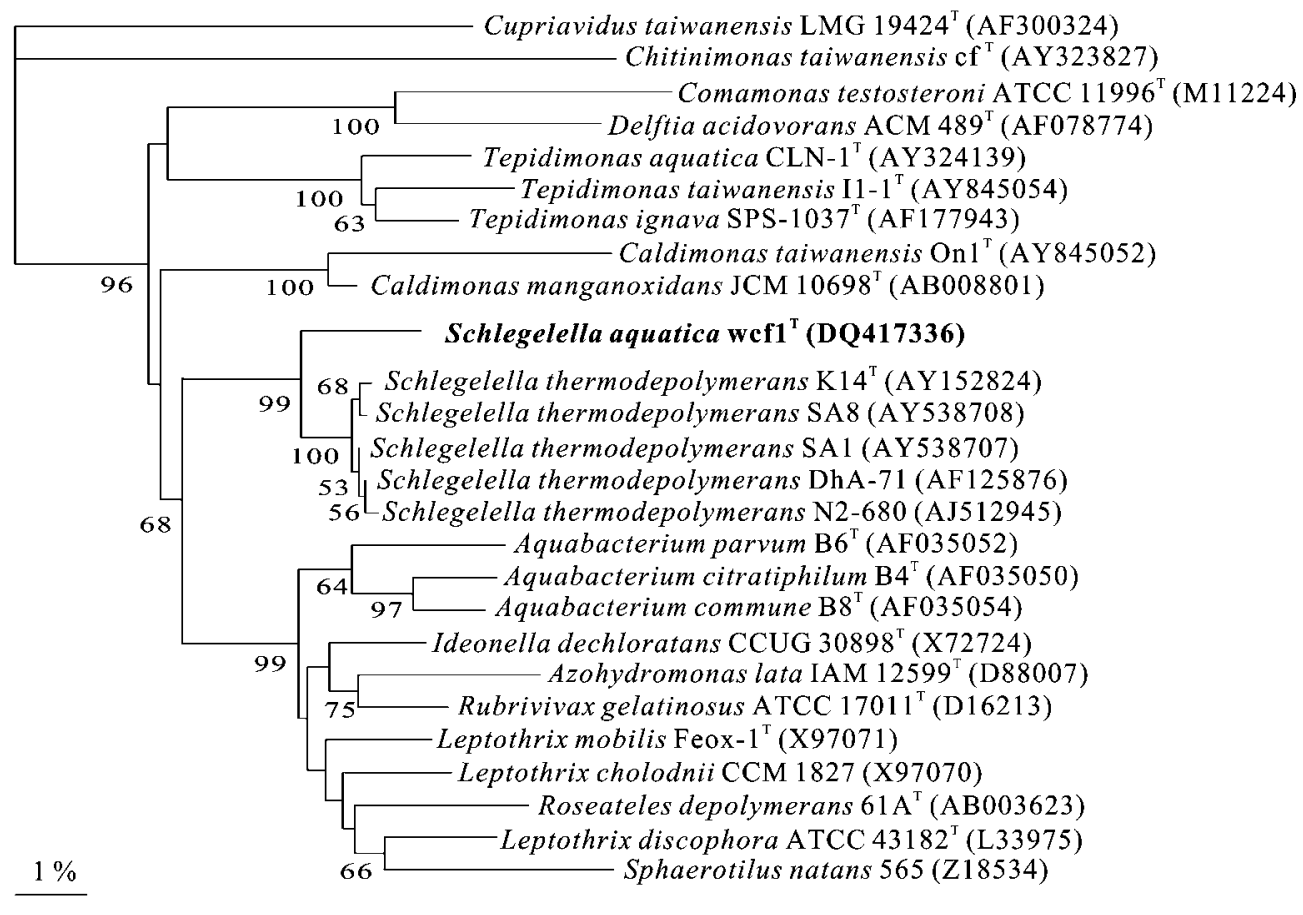

Fig. 1. Phylogenetic analysis based on 16S rRNA gene sequences available from GenBank/EMBL/DDBJ (accession numbers are given in parentheses) constructed after multiple alignments of data showing the position of strain wcf $1^{\top}$ in the genus Schlegelella. Distances and clustering with the neighbour-joining method were performed using BioEdit. Numbers at nodes are percentage bootstrap values based on 1000 resampled datasets; only values above $50 \%$ are given. The sequence of Cupriavidus taiwanensis was used as an outgroup. Bar, $1 \%$ sequence dissimilarity per nucleotide position.

Table 1. Comparison of long-chain fatty acid compositions (\%) of S. aquatica sp. nov. strain $\mathrm{wcf}^{\top}{ }^{\top}$ and S. thermodepolymerans $\mathrm{K} 14^{\mathrm{T}}$

Data for $S$. thermodepolymerans $\mathrm{K} 14^{\mathrm{T}}$ are from Elbanna et al. (2003). Strain $\mathrm{wcfl}^{\mathrm{T}}$ was cultivated for $48 \mathrm{~h}$ on TSA medium (Difco) at $50^{\circ} \mathrm{C}$, similar to the conditions described for S. thermodepolymerans $\mathrm{K} 14^{\mathrm{T}}$ by Elbanna et al. (2003). -, Fatty acid values of less than $1 \%$; ND, not detected.

\begin{tabular}{|lcc|}
\hline Fatty acid & Strain $_{\mathbf{w c f 1}}{ }^{\mathbf{T}}$ S. thermodepolymerans $\mathbf{K 1 4}^{\mathbf{T}}$ \\
\hline $10: 0$ & $1 \cdot 2$ & $1 \cdot 3$ \\
$10: 0$ 3-OH & $5 \cdot 1$ & $3 \cdot 3$ \\
$12: 0$ & $2 \cdot 3$ & $1 \cdot 6$ \\
$12: 03-\mathrm{OH}$ & $\mathrm{ND}$ & $2 \cdot 0$ \\
$16: 0$ & $48 \cdot 7$ & $43 \cdot 1$ \\
$16: 0$ iso & $\mathrm{ND}$ & - \\
$17: 0$ cyclo & $16 \cdot 5$ & $32 \cdot 6$ \\
$18: 1 \omega 7 c$ & $4 \cdot 3$ & $4 \cdot 4$ \\
$18: 1$ & - & - \\
$18: 0$ & $1 \cdot 0$ & - \\
Summed feature $3^{*}$ & $19 \cdot 5$ & - \\
& & \\
\hline
\end{tabular}

${ }^{*}$ Summed feature 3 comprises $16: 1 \omega 7 c$ and/or 15:0 iso $2-\mathrm{OH}$. arylamidase, trypsin, $\alpha$-chymotrypsin, acid phosphatase, $\alpha$ galactosidase, $\beta$-galactosidase, $\beta$-glucuronidase, $\beta$-glucosidase, $N$-acetyl- $\beta$-glucosaminidase, $\alpha$-mannosidase and $\alpha$-fucosidase. The following compounds were utilized as sole carbon sources in the Biolog GN2 test system: glycogen, methyl pyruvate, monomethyl succinate, acetic acid, $\alpha$ hydroxybutyric acid, $\beta$-hydroxybutyric acid, $\rho$-hydroxyphenylacetic acid, $\alpha$-ketobutyric acid, $\alpha$-ketoglutaric acid, DL-lactate, propionic acid, succinic acid, succinamic acid, Lalaninamide, D-alanine, L-alanine, L-asparagine, L-proline, L-pyroglutamic acid and urocanic acid. However, strain wcf $^{\mathrm{T}}$ could not oxidize $\alpha$-cyclodextrin, dextrin, Tween 40 , Tween 80, $N$-acetyl-D-galactosamine, $N$-acetyl-D-glucosamine, adonitol, arabinose, arabitol, cellobiose, i-erythritol, D-fructose, L-fucose, D-galactose, gentiobiose, myo-inositol, $\alpha$-D-lactose, lactulose, D-mannitol, D-mannose, melibiose, methyl $\beta$-D-glucoside, D-psicose, D-raffinose, L-rhamnose, D-sorbitol, sucrose, D-trehalose, turanose, xylitol, cisaconitic acid, citrate, formic acid, D-galactonic acid lactone, D-galacturonic acid, D-glucosaminic acid, D-glucuronic acid, $\gamma$-hydroxybutyric acid, itaconic acid, $\alpha$-ketovaleric acid, malonic acid, quinic acid, D-saccharic acid, sebacic acid, bromosuccinic acid, glucuronamide, L-alanyl glycine, L-aspartic acid, L-glutamic acid, glycyl L-aspartic acid, glycyl 
L-glutamic acid, L-histidine, hydroxy-L-proline, L-leucine, L-ornithine, L-phenylalanine, D-serine, L-serine, L-threonine, DL-carnitine, $\gamma$-aminobutyric acid, inosine, uridine, thymidine, phenylethylamine, putrescine, 2-aminoethanol, 2,3-butanediol, glycerol, DL- $\alpha$-glycerol phosphate, D-glucose 6-phosphate and $\alpha$-D-glucose 1-phosphate.

Strain $\mathrm{wcfl}^{\mathrm{T}}$ was susceptible to ampicillin, cefotaxime, chloramphenicol, gentamicin, kanamycin, nalidixic acid, novobiocin, penicillin $G$, rifampicin, streptomycin and tetracycline.

Further comparisons of physiological and biochemical characteristics between strain $\mathrm{wcfl}^{\mathrm{T}}$ and $S$. thermodepolymerans $\mathrm{K} 14^{\mathrm{T}}$ are presented in Table 2. It is apparent from Table 2 that strain $\mathrm{wcfl}^{\mathrm{T}}$ could be distinguished from $S$. thermodepolymerans $\mathrm{K} 14^{\mathrm{T}}$ using a combination of phenotypic attributes (nitrate reduction, assimilation of carbon substrates such as arabinose, adipate, citrate, maltose and phenyl acetate and some enzyme activities such as catalase, cystine arylamidase and $\alpha$-glucosidase). Despite physiological and biochemical attributes, the results of fatty acid composition and DNA-DNA hybridization clearly distinguished strain $\mathrm{wcfl}^{\mathrm{T}}$ from $S$. thermodepolymerans $\mathrm{K} 14^{\mathrm{T}}$. On the basis of the results of this polyphasic taxonomic study, strain $\mathrm{wcfl}^{\mathrm{T}}$ represents a novel species of the genus Schlegelella for which the name Schlegelella aquatica sp. nov. is proposed.

\section{Description of Schlegelella aquatica sp. nov.}

Schlegelella aquatica (a.qua'ti.ca. L. fem. adj. aquatica living in water).

Cells are Gram-negative, non-spore-forming, rod-shaped, $0 \cdot 4-0 \cdot 5 \mu \mathrm{m}$ in diameter and $0 \cdot 8-2 \cdot 0 \mu \mathrm{m}$ in length. Cells occur singly and are motile with a single polar flagellum. Growth is evident at temperatures of $30-60{ }^{\circ} \mathrm{C}$ and $\mathrm{pH} 6 \cdot 0-8 \cdot 0$. Optimum growth is at $50^{\circ} \mathrm{C}$ and $\mathrm{pH} 7 \cdot 0$. Positive for nitrate reduction, gelatin hydrolysis and aesculin hydrolysis and weakly positive for oxidase activity. Assimilates glucose, maltose, gluconate, adipate and phenyl acetate. Major fatty acids are 16:0, 17:0 cyclo and summed feature $3(16: 1 \omega 7 c$ and/or $15: 0$ iso $2-\mathrm{OH})$. Additional biochemical characteristics are listed above and in Table 2.

Table 2. Phenotypic characteristics that differentiate S. aquatica sp. nov. strain $\mathrm{wcf}^{\top}{ }^{\top}$ from $S$. thermodepolymerans $\mathrm{K} 14^{\top}$

Data for S. thermodepolymerans $\mathrm{K} 14^{\mathrm{T}}$ were acquired in the present study, except for the data on DNA G+C content (Elbanna et al., 2003). + , Positive; -, negative; W, weak reaction; R, resistant; S, sensitive. Both strains are positive for C4 esterase, C8 esterase lipase, leucine arylamidase, naphthol-AS-BI-phosphohydrolase and assimilation of gluconate, lactate, acetate, succinate and pyruvate. The following characteristics are negative for both strains: trypsin, $\alpha$-chymotrypsin, $\alpha$-galactosidase, $\beta$-galactosidase, $\beta$-glucuronidase, $\alpha$-glucosidase, $\beta$-glucosidase, $N$-acetyl- $\beta$-glucosaminidase, $\alpha$-fucosidase, $\alpha$-mannosidase and assimilation of mannose, galactose, fructose, rhamnose, sucrose, trehalose, mannitol, inositol, erythritol, adonitol and xylitol.

\begin{tabular}{|c|c|c|}
\hline Characteristic & Strain $\mathrm{wcfl}^{\mathrm{T}}$ & S. thermodepolymerans $\mathrm{K} 14^{\mathrm{T}}$ \\
\hline Oxidase & $\mathrm{W}$ & + \\
\hline Catalase & - & + \\
\hline Cystine arylamidase & - & + \\
\hline$\alpha$-Glucosidase & + & - \\
\hline Nitrate reduction & + & - \\
\hline \multicolumn{3}{|l|}{ Assimilation of: } \\
\hline Arabinose & - & + \\
\hline Maltose & + & - \\
\hline Adipate & + & - \\
\hline Citrate & - & + \\
\hline Phenyl acetate & + & - \\
\hline L-Glutamic acid & - & + \\
\hline \multicolumn{3}{|l|}{ Susceptibility to: } \\
\hline Penicillin & S & $\mathrm{R}$ \\
\hline Ampicillin & S & $\mathrm{R}$ \\
\hline Streptomycin & $\mathrm{S}$ & $\mathrm{R}$ \\
\hline Rifampicin & S & $\mathrm{R}$ \\
\hline Maximum temperature for growth $\left({ }^{\circ} \mathrm{C}\right)^{\star}$ & 60 & 65 \\
\hline Optimum growth temperature $\left({ }^{\circ} \mathrm{C}\right)^{*}$ & 50 & $50-55$ \\
\hline Isolation source & Hot spring & Sludge \\
\hline DNA $\mathrm{G}+\mathrm{C}$ content $(\mathrm{mol} \%)$ & $69 \cdot 2$ & $70 \cdot 0$ \\
\hline
\end{tabular}

${ }^{\star}$ Strains $\mathrm{K} 14^{\mathrm{T}}$ and $\mathrm{wcfl}^{\mathrm{T}}$ were inoculated into $50 \mathrm{ml} 10 \% \mathrm{LB}$ broth in a $250 \mathrm{ml}$ flask and agitated at 125 r.p.m. for $48 \mathrm{~h}$. 
The DNA G + C content of the type strain is $69 \cdot 2 \mathrm{~mol} \%$. The type strain, $\operatorname{wcfl}^{\mathrm{T}}\left(=\mathrm{BCRC} 17557^{\mathrm{T}}=\mathrm{LMG} 23380^{\mathrm{T}}\right)$, was isolated from a water sample from a hot spring in Guanzingling located in the Tainan area of southern Taiwan.

\section{References}

Chen, W.-M., Laevens, S., Lee, T.-M., Coenye, T., de Vos, P., Mergeay, M. \& Vandamme, P. (2001). Ralstonia taiwanensis sp. nov., isolated from root nodules of Mimosa species and sputum of a cystic fibrosis patient. Int J Syst Evol Microbiol 51, 1729-1735.

Chung, Y. C., Kobayashi, T., Kanai, H., Akiba, T. \& Kudo, T. (1995). Purification and properties of extracellular amylase from the hyperthermophilic archeon Thermococccus profundus DT5432. Appl Environ Microbiol 61, 1502-1506.

Elbanna, K., Lütke-Eversloh, T., van Trappen, S., Mergaert, J., Swings, J. \& Steinbüchel, A. (2003). Schlegelella thermodepolymerans gen. nov., sp. nov., a novel thermophilic bacterium that degrades poly(3-hydroxybutyrate-co-3-mercaptopropionate). Int J Syst Evol Microbiol 53, 1165-1168.

Ezaki, T., Hashimoto, Y. \& Yabuuchi, E. (1989). Fluorometric DNADNA hybridization in microdilution wells as an alternative to membrane filter hybridization in which radioisotopes are used to determine genetic relatedness among bacterial strains. Int J Syst Bacteriol 39, 224-229.
Hall, T. A. (1999). BioEdit: a user-friendly biological sequence alignment editor and analysis program for Windows 95/98/NT. Nucleic Acids Symp Ser 41, 95-98.

Jukes, T. H. \& Cantor, C. R. (1969). Evolution of protein molecules. In Mammalian Protein Metabolism, vol. 3, pp. 21-132. Edited by H. N. Munro. New York: Academic Press.

Lütke-Eversloh, T., Elbanna, K., Cnockaert, M. C., Mergaert, J., Swings, J., Manaia, C. M. \& Steinbüchel, A. (2004). Caenibacterium thermophilum is a later synonym of Schlegelella thermodepolymerans. Int J Syst Evol Microbiol 54, 1933-1935.

Manaia, C. M., Nunes, O. C. \& Nogales, B. (2003). Caenibacterium thermophilum gen. nov., sp. nov., isolated from a thermophilic aerobic digester of municipal sludge. Int J Syst Evol Microbiol 53, 1375-1382.

Mesbah, M., Premachandran, U. \& Whitman, W. B. (1989). Precise measurement of the $\mathrm{G}+\mathrm{C}$ content of deoxyribonucleic acid by highperformance liquid chromatography. Int J Syst Bacteriol 39, 159-167.

Romen, F., Reinhardt, S. \& Jendrossek, D. (2004). Thermotolerant poly(3-hydroxybutyrate)-degrading bacteria from hot compost and characterization of the PHB depolymerase of Schlegelella sp. KB1a. Arch Microbiol 182, 157-164.

Saitou, N. \& Nei, M. (1987). The neighbor-joining method: a new method for reconstructing phylogenetic trees. Mol Biol Evol 4, 406-425.

Yu, Z. \& Mohn, M. M. (1999). Isolation and characterization of thermophilic bacteria capable of degrading dehydroabietic acid. Can J Microbiol 45, 513-519. 